\title{
Integrated Movable Micromechanical Structures for Sensors and Actuators
}

\author{
LONG-SHENG FAN, MEMBER, IEEE, YU-CHONG TAI, AND RICHARD S. MULLER, FELlOw, IEEE
}

\begin{abstract}
Movable pin joints, gears, springs, cranks, and slider structures with dimensions measured in micrometers have been fabricated using silicon microfabrication technology. These micromechanical structures, which have important transducer applications, are batch-fabricated in an IC-compatible process. The movable mechanical elements are built on layers that are later removed so that they are freed for translation and rotation. A new undercut-and-refill technique that makes use of the high surface mobility of silicon atoms undergoing chemical vapor deposition is used to refill undercut regions in order to form restraining flanges. Typical element sizes and masses are measured in millionths of a meter and billionths of a gram. The process provides the tiny structures in an assembled form, avoiding the nearly impossible challenge of handling such small elements individually.
\end{abstract}

\section{INTRODUCTION}

$\mathrm{T}_{\mathrm{t}}$ HE UNPRECEDENTED growth of integrated-circuit technology and computing techniques has made sophisticated data processing accurate, economical, and widely available. Today's electronic systems are capable of dealing with large numbers of physical input and output variables, but the transducers that provide interfaces between the electrical and physical world are in many cases outmoded and dependent on awkward hybrid-fabrication techniques. Many of the materials and processes used to produce integrated microcircuits, however, can be employed in new ways to produce microsensors and actuators. These structures complement the IC process and provide a means to produce new electronic systems.

Thus far, micromechanical transducer structures such as cantilevers, bridges, and diaphragms have been fabricated with IC-compatible processes for various useful applications. These structures, however, contain only bendable joints, a severe limitation on mechanical design capabilities for many applications. Microstructures with rotatable joints, sliding and translating members, and mechanical-energy storage elements would provide the basis for a more general micromechanical transducer-system design. Because such structures add important degrees of freedom to designers, we have investigated techniques to fabricate them using IC-based microfabrication processes [1]. Rotatable silicon elements, made using IC technol-

Manuscript received October 1, 1987; revised January 19, 1988. The Berkeley Sensor and Actuator Center is an NSF/Industry/University Cooperative Research Center. This work was partially supported by the U.S. Army Harry Diamond Laboratory.

The authors are with the Berkeley Sensor and Actuator Center, Department of Electrical Engineering and Computer Sciences, and the Electronics Research Laboratory, University of California, Berkeley, CA 94720.

IEEE Log Number 8820452. ogy, have also been reported by Gabriel et al. [2]. The new mechanical elements use polysilicon thin-film technology combined with techniques that we describe in this paper. An important advantage of the procedures described is that they provide mechanical structures containing more than one part in a preassembled form; this avoids individually handling the very tiny structures. The initial demonstration of the technique to make these structures employs polysilicon as the structural material and phosphosilicate glass for the sacrificial layer. Other materials may, however, be used in place of these, provided that they are compatible with the overall process.

\section{Structures and Processes}

\section{A. Fixed-Axle Pin Joints}

A pin joint is composed of an axle around which a member (rotor) is free to rotate. Movement along the axle by the rotor is constrained by flanges. Fig. 1 shows the cross section and top view of a pin joint fixed to the substrate that has been fabricated using polycrystalline silicon. The rotor, axle, and flange are all made of polysilicon that has been deposited by a low-pressure chemicalvapor-deposition (LPCVD) process on top of a silicon substrate. The pin joint is produced using a double-polysilicon process and a phosphosilicate glass (PSG) sacrificial layer in a three-mask process as indicated in Fig. 2. In this process, openings are first made by dry etching a composite layer of polysilicon on PSG deposited by sequential LPCVD processes. Another PSG layer is deposited over the entire structure including the edges of the circular openings. Photolithography steps are then used to expose bare silicon at specific locations so that a subsequent deposition of polysilicon will anchor to the silicon substrate at these desired places. After depositing and patterning the second polysilicon layer for axles and flanges, all previously deposited PSG layers are removed in buffered hydrofluoric acid (BHF). The remaining polysilicon layers form the pin-joint structure. The rotor is free to rotate when the PSG layer is removed by BHF. An SEM photo (Fig. 3) shows a completed pin joint of this type.

\section{B. Self-Constraining Pin Joints}

A rotating-joint structure that provides several new possibilities for mechanical design can be built using only a small variation on the process described above. To differentiate joints of this type from the fixed-axle pin joints 


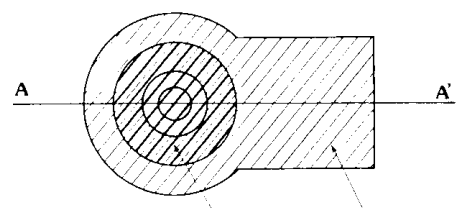

2nd Polysilicon 1st Polysilicon

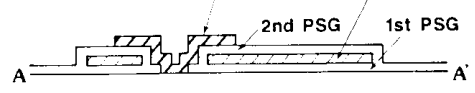

Fig. 1. Top view and cross section of a polysilicon micromechanical pin joint.
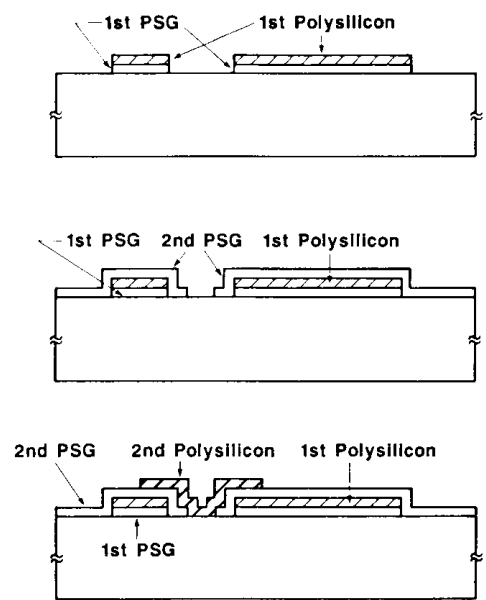

Fig. 2. Fabrication process for the anchored pin joint shown in Fig. 1.

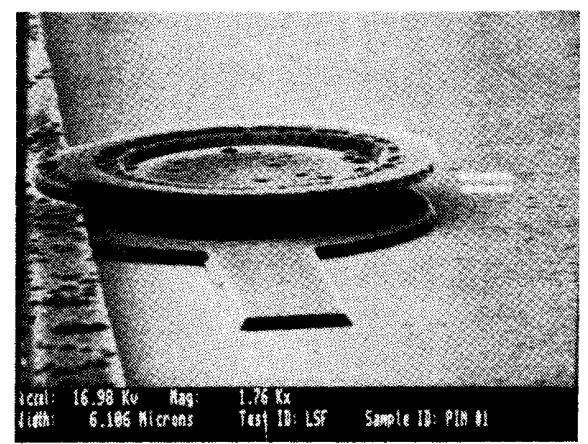

Fig. 3. SEM photograph of anchored pin joint. The outer radius of the flange connected to the axle is $25 \mu \mathrm{m}$.

described above, we call these structures self-constraining joints. Self-constraining joints can, for example, allow for rotation while, at the same time, permitting translation across the silicon surface. These joints need to have a flange on the axle underneath the rotor to keep it in place. The axle can either be fixed to the substrate or else left free to translate across its surface.

Self-constraining joints are produced by a double polysilicon process with a PSG sacrificial layer. An undercutand-refill technique is introduced to position the second-

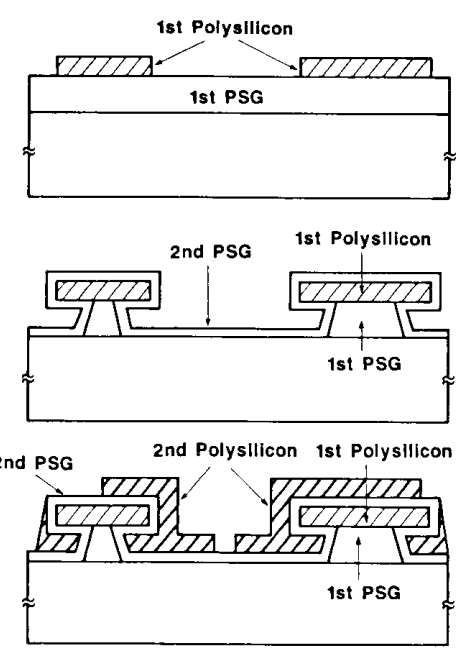

Fig. 4. Fabrication process for the self-constraining joint.

layer polysilicon both over and under the axle formed of first-layer polysilicon. Fig. 4 outlines the process for these joints, which are produced using two masks. In this process, after the PSG and polysilicon layers have been deposited by LPCVD, the polysilicon is patterned by dry etching. The next step is to use a timed etch of the first PSG layer to undercut the polysilicon. An optional mask may be used if only selected regions are to be undercut. Another PSG layer is then deposited. A second polysilicon layer that fills in the undercut regions is the patterned to produce axles and flanges. After this, all PSG layers are removed in a buffered hydrofluoric-acid solution. The remaining polysilicon layers form the self-constraining joint structure as shown in the SEM photograph of Fig. 5. The interleaved polysilicon layers, evident in Fig. 4, can be made because of the high surface mobility of silicon atoms during the LPCVD process. This permits the undercut regions to refill so that restraining flanges remain over and under the first-layer polysilicon.

For more complex structures, both pin joints and selfconstrained joints can be made in the same process. Fig. 6 shows four-joint crank structures fabricated using both types of joints in a three-mask process (four, if the optional undercut mask is used). In Fig. 6, the joints at both ends of the central element are self-constraining but they are freed from the substrate. The other two joints are pinned to the substrate. Note that, except for the fixed joints, the entire structure has moved from its original position, indicated by a darkened pattern on the silicon substrate in Fig. 6. Using a surface profiler (Alpha-Step 200), we have found that in the darkened pattern there is a pit that is roughly $100 \mathrm{~nm}$ deep. This pit appears to be caused by enhanced etching of the silicon surface by the BHF under the polysilicon moving elements. The enhanced etching may, in turn, result from localized stress in this region. Further research to test this hypothesis is underway. 


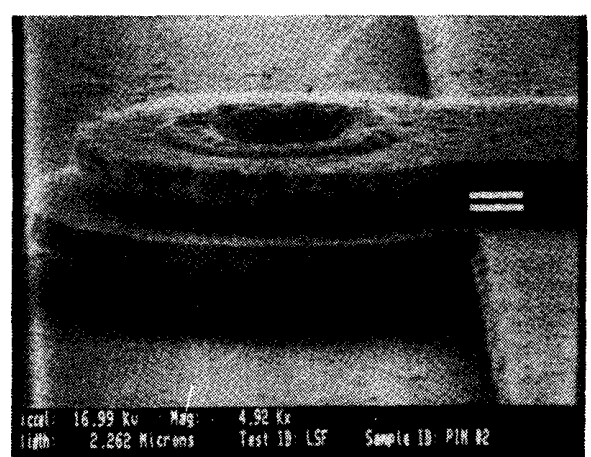

Fig. 5. SEM photograph of a self-constraining joint. The rotor is attached to a hub that turns in a collar projecting from a stationary polysilicon surface. There is a retention flange on the hub below the collar.

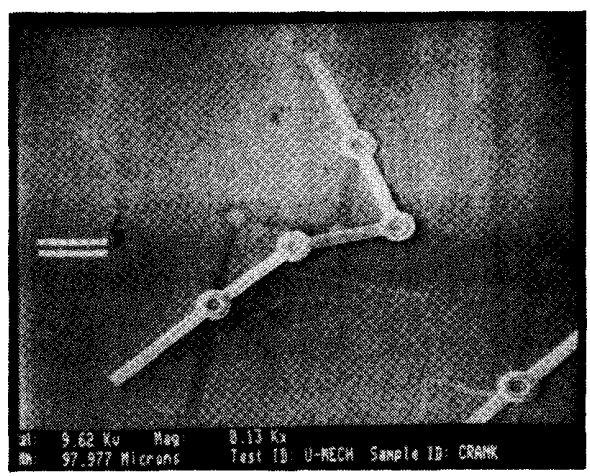

Fig. 6. SEM photograph of a four-joint crank having a central arm held by self-constraining joints that are free to translate. The original position of the crank is indicated by the darker pattern. All crank arms are 150 $\mu \mathrm{m}$ in length.

\section{Flanged Structures}

The procedures carried out to make the two types of pin joints can be employed to produce other mechanical structures. For example, the three-mask pin-joint process can be used directly to fabricate the square slider shown in Fig. 7. The slider has a polysilicon moving element that is constrained by flanges along two of its edges so that only translational movement in one dimension is allowed. In the gear-slider combination of Fig. 8 (produced by the pin-joint process with four masks), the slider has a guide at its center to constrain movement to one dimension. Fig. 9 shows a crank-slot combination that requires five masks to produce. The slot element, formed by first-layer polysilicon, is pinned to the substrate by another element made of second-layer polysilicon so that both translational and rotational movements can take place. The joint for the crank-slot in Fig. 9 is self-constraining.

\section{Design Variations}

The versatility of the techniques described permits many potentially useful variations in design. As an example, Fig. 10 is a slider that can be thought of as a dual to the structure in Fig. 7. The flanges holding the parts together

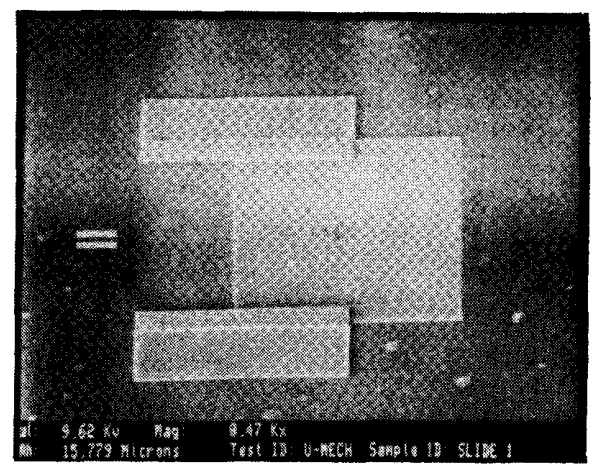

Fig. 7. Square slider with two edges restricted by flanges. One side of the slider is $100 \mu \mathrm{m}$ and the central opening is a square of $10 \mu \mathrm{m}$.

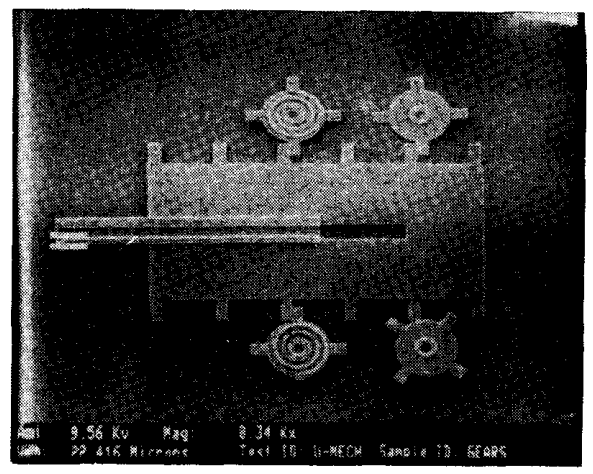

Fig. 8. Gear and slider combination. The slider is 210 by $100 \mu \mathrm{m}$. The toothed edges mesh with four gears, two of which have flat spiral springs attached.

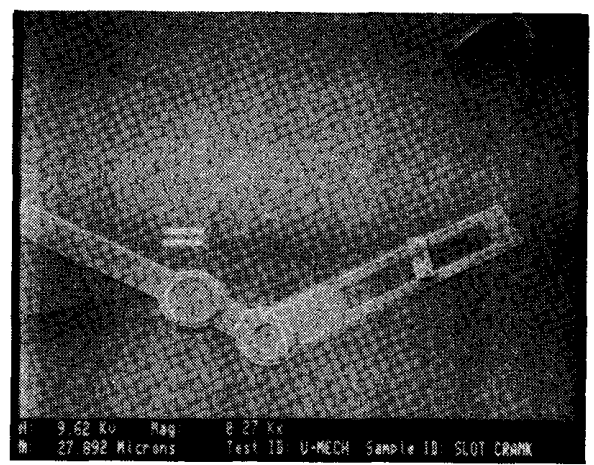

Fig. 9. Crank-slot combination with a center pin. The slot is $130 \mu \mathrm{m}$ long and $20 \mu \mathrm{m}$ wide. The diameters of the two joints are each $50 \mu \mathrm{m}$.

are on opposite members in each of these two versions of the mechanical slider. In the same sense, Fig. 11 shows a four-joint crank that is dual to the one in Fig. 6. All four joints in this crank are made using a self-constraining process; the two end joints are fixed and the two center joints can translate.

\section{E. Micromechanical Bushings}

The undercut-and-refill technique is useful for other applications also. When combined with pin joints, this tech- 


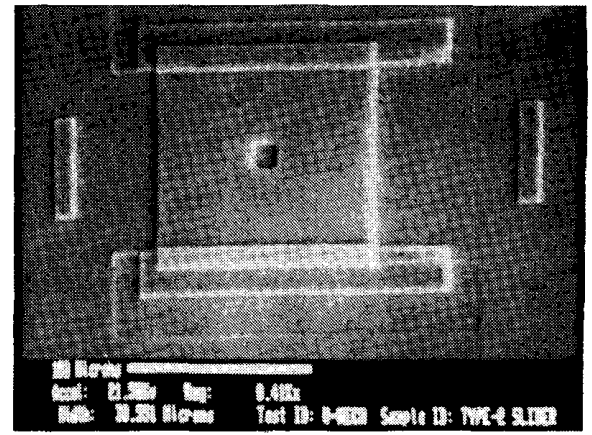

Fig. 10. Slider structure with outer edges guided by self-constraining joints. Stops limit the extent of lateral motion by the slider.

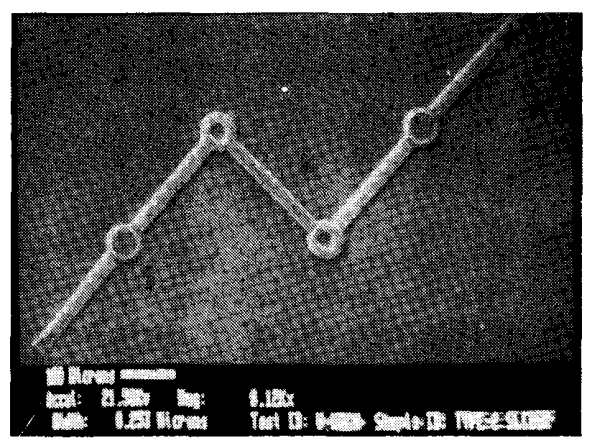

Fig. 11. Four-joint crank made with self-constraining joints.

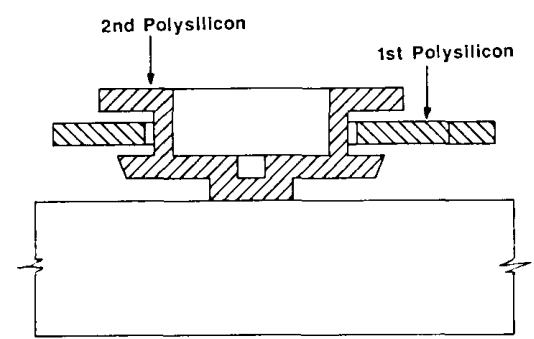

Fig. 12. Cross section of a micromechanical bushing built by the self-constraining-joint process.

nique permits the fabrication of bushings that can be used, for example, to elevate a rotor away from the silicon surface. This can greatly reduce frictional forces, especially if the bushing elements are coated with or made from another material such as silicon nitride that may provide better wear properties. Fig. 12 shows a cross section for a bushing produced using the self-constraining-joint process described above and one extra mask for anchoring to the substrate (three masks in all).

\section{F. Polysilicon Springs}

Mechanical energy storage is important in many systems, and therefore it is very desirable to be able to fabricate micromechanical springs. These elements can also be produced using the process described above. The flat spiral spring attached to a pin joint, shown in Fig. 13, is

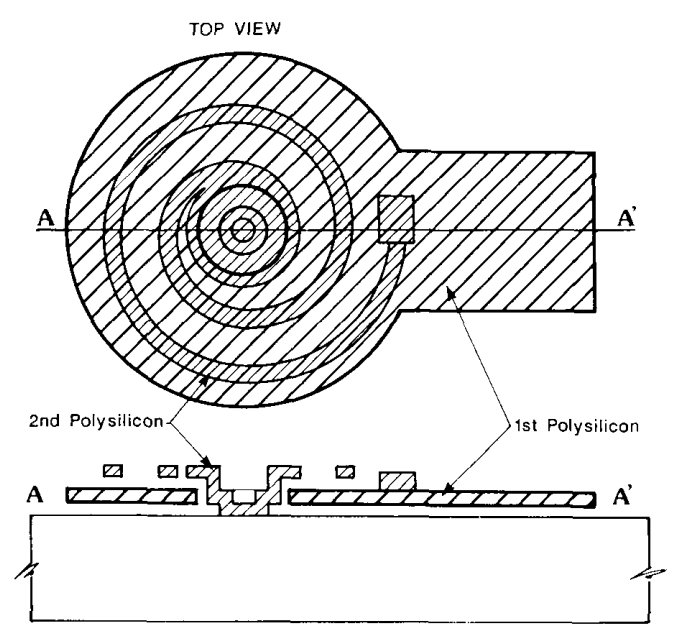

CROSS SECTION

Fig. 13. Top view and cross section of flat spiral spring fixed on one end to an axle.

made of second-layer polysilicon and connected on one end to the axle of a pin joint. The other end is attached to a movable disc made of first-layer polysilicon. The spring, produced using four masks, returns the disc to its original position after it is displaced. Figs. 14 and 15 show SEM photographs of restraining springs connecting rotors to pin-joint axles. Both springs are made of $2-\mu \mathrm{m}$-wide second-layer polysilicon. Shown in Fig. 15 is a beam spring that has an appreciably larger spring constant than does the flat spiral spring of Fig. 14.

\section{G. Processing Details}

In the foregoing, we have described the essential techniques for the in situ fabrication of assembled micro structures. The achievable dimensions for the finished elements depend on the lithography and processing steps used, but they can be roughly estimated at ten or fewer micrometers. Any of the microstructures can be fabricated separately using fewer than four masks. Six masks are needed to build all of the structures in the same run. When all are produced at one time there is an unavoidable loss in element precision because of the extra processing steps.

To illustrate the processes more completely, we describe in fuller detail the steps used to fabricate all structures on the same chip. First, a 1.5- $\mu$ m-thick phosphorusdoped ( 8 wt. \%) LPCVD silicon-dioxide layer is deposited at $450^{\circ} \mathrm{C}$ on a (100) silicon substrate. Photolithography and the first mask are used to open selected areas on the substrate where the first-layer polysilicon is to be anchored. Undoped LPCVD polysilicon, $1.5 \mu \mathrm{m}$-thick, is then deposited at $630^{\circ} \mathrm{C}$ and patterned with a second mask in a $\mathrm{CCl}_{4}$ plasma. The third mask is used to define the undercut regions for self-constraining and bushing structures. Buffered HF etching creates a $2-\mu \mathrm{m}$ undercut. Next, a $0.5-\mu$ m-thick phosphorus-doped ( $8 \mathrm{wt} . \%)$ LPCVD sil- 


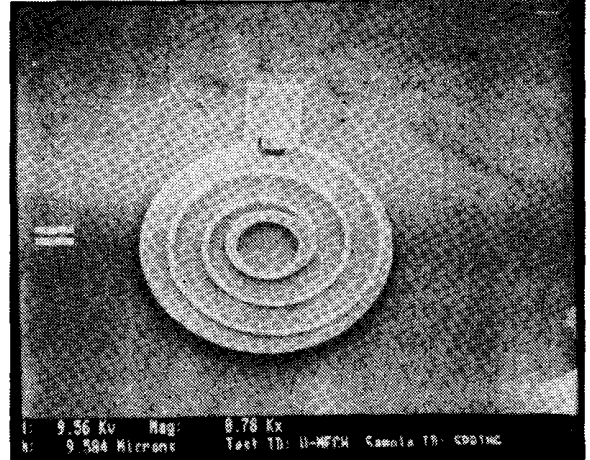

Fig. 14. SEM photograph of the spring-axle structure shown in Fig. 13 The 2.5-revolution spiral spring is made of $2-\mu \mathrm{m}$-wide second-layer polysilicon. Its inner end is fixed to an axle $10 \mu \mathrm{m}$ in diameter, and its outer end is connected to a movable arm.

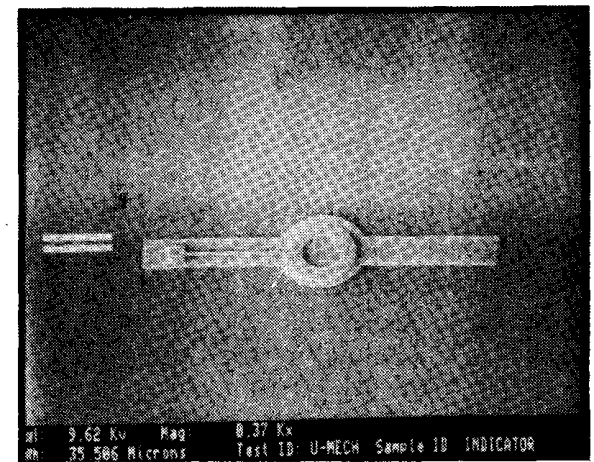

Fig. 15. SEM photograph of a beam spring attached to a central axle. The beam is $60 \mu \mathrm{m}$ long and $2 \mu \mathrm{m}$ wide.

icon-dioxide sacrificial layer is deposited at $450^{\circ} \mathrm{C}$. The fourth and fifth masks are used to pattern the silicon-dioxide layer to anchor the spring element to the substrate and the rotor, respectively. Undoped LPCVD polysilicon, 1.0 $\mu \mathrm{m}$ thick, is then deposited at $630^{\circ} \mathrm{C}$, defined and patterned using the sixth mask, and eteched in a $\mathrm{CCl}_{4}$ plasma. Prolonged etching (and therefore thick resist films) are required to remove completely any residue of polysilicon from the regions near to topographic steps. For shorter etching times, a more isotropic plasma such as $\mathbf{S F}_{6}$ might be used. A 1-h annealing step in nitrogen at $1000^{\circ} \mathrm{C}$ is used to reduce stress in the polysilicon. To release the structures from the oxide required $6 \mathrm{~h}$ of etching in a $5: 1$ buffered HF solution.

\section{Study of Mechanical Properties}

An important use for these structures is to carry out research on the micromechanical properties of materials. This research is especially necessary since many of the materials have thus far been applied exclusively for electronics. One means for obtaining useful data is to carry out visual inspection of high-speed magnified video-tape images that show the response of dynamically actuated elements. Analysis of these data will permit studies of

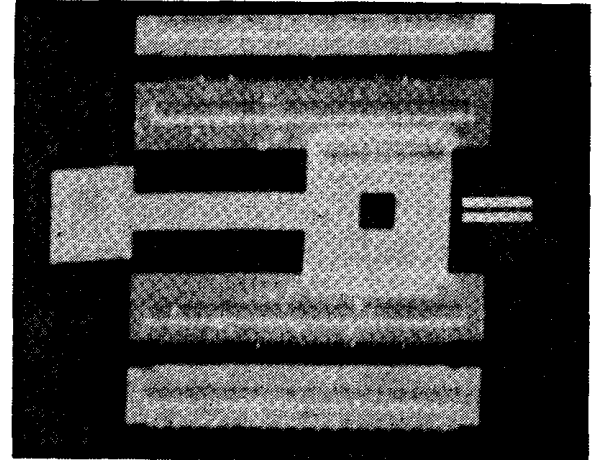

Fig. 16. Slider and bridge structure. One end of the bridge is anchored to the substrate and the other is attached to a slider. The central square opening is $20 \mu \mathrm{m}$ on a side and the square slider is $80 \mu \mathrm{m}$ on a side.

frictional behavior, damping, fatique limits, and of fundamental properties, such as Young's modulus, Poisson's ratio, and the orientational dependences of these parameters.

In general, a residual stress is found in LPCVD polysilicon after deposition. Previous papers [3], [4] have described useful ways to study uniform stress distributions. The stress distribution in the direction of polysilicon film growth is, however, very likely not to be uniform and therefore to induce a bending moment across the films. The bending moment is of special concern in cases where flatness is important.

Using slider structures (Figs. 7 and 10), we expect to be able to separate the effects of uniform and bending stresses in thin-film mechanical structures. Fig. 16 shows the top view of a slider and a bridging beam. The outer edge of the flanges are defined with teeth to act as measuring scales. One end of the beam is anchored on the silicon substrate, and the other end is connected to a selfconstraining slider. Since the slider allows translational movement, the compressive-stress component in the polysilicon beam can be released after freeing the whole structure, while the bending moment will be left in the beam. Detailed analytical study of such structures to determine both the compressive stress and the bending moment in deposited polysilicon is underway.

\section{Fracture-Strength Study}

A flat spiral spring made of second-layer polysilicon is used to restrain a pin joint. Within its fracture limit, the spring can return the structure to its original position after it has been moved. Experiments have been done on these spring structures to estimate the lateral fracture stength of the polysilicon. A simplified mechanical analysis provides the basis for this estimate. For a $2 \mu \mathrm{m}$-wide $1-\mu \mathrm{m}$ thick spring extending 2.5 revolutions with inner radius $r_{1}=10 \mu \mathrm{m}$ connected to the central axle and outer radius $r_{2}=30 \mu \mathrm{m}$ connected to one arm, fractures occur at deflections of roughly $300^{\circ}$. For a spiral spring of width $h$ and thickness $t$, assuming that adjacent turns do not come 
into contact, the energy stored is [5]

$$
U=\int_{0}^{l} \frac{M^{2} d s}{2 E_{Y} I}
$$

where $M$ is the bending moment, $E_{Y}$ is Young's modulus, $I$ is the moment of inertia of the spring cross section, $d s$ is the length of a small element of the spring, and $l$ is the total length of the spiral. The spiral is generated using the equation $r=a \theta$ where $\theta$ is the polar-coordinate angle and $a$ is a design constant chosen for a particular spring size. The bending moment can be shown [5] to be constant along the length of the spring. The angular deflection $\Phi$ is

$$
\Phi=\frac{\partial U}{\partial M}=\int_{0}^{l} \frac{M}{E_{Y} I} d s=\frac{M}{E_{Y} I} \frac{r_{2}^{2}-r_{1}^{2}}{2 a} .
$$

The moment of inertia of the rectangular cross section is $I=t h^{3} / 12$ and the maximum stress in the spring $\sigma_{\max }$ is $M h / 2 I$. Using these equivalents in (2), we can express the maximum stress in terms of the angular deflection $\Phi$.

$$
\sigma_{\max }=\frac{E_{Y} h a}{r_{2}^{2}-r_{l}^{2}} \Phi
$$

The spiral springs were unwound using microprobes until they fractured. The bending moment in the spring loaded in this manner is constant along its length. Spring fractures occurred in all cases at deflection angles of 300 $\pm 30^{\circ}$. The fractures were observed in one or several locations and typically more then $20 \mu \mathrm{m}$ away from the attachment points. The spiral springs (of the type shown in Fig. 13), have inner and outer radii of $r_{1}=10 \mu \mathrm{m}$ and $r_{2}$ $=30 \mu \mathrm{m}$, respectively. Other parameters are: thickness $t$ $=1 \mu \mathrm{m}$, width $h=2 \mu \mathrm{m}$, and spiral constant $a=1.27$ $\mu \mathrm{m}$. Using the observed value of $\Phi_{\text {fract }}$ at fracture $\left(300^{\circ}\right.$ or 5.24 radians) in (3), we calculate a fracture strength $\sigma_{\text {tract }}$ that is 1.7 percent times Young's modulus $E_{Y}$ for thin-film polysilicon. At least two simplifying assumptions underlie the conclusions made above: 1) that the spring motion is entirely in the horizontal plane (neglecting possible vertical motions that would relax stress), and 2) that the spring has sufficient turns to be treated as ideal [6]. Other studies, still in progress, indicate a slightly lower fracture strength (in the order of 1.3-1.4 percent of $E_{Y}$ ). For a perspective on our results, we note that the highest reported fracture strength for single-crystal silicon is 2.6 percent times $E_{Y}[7]$. We expect that values for $E_{Y}$ will depend on the deposition conditions for the film and on the direction of the stress relative to the growth direction.

To estimate $E_{Y}$ for our polysilicon films, we make use of Johnson's analysis [8], X-ray diffraction studies showing the distribution of crystalline orientations in our films, and published orientation-dependent elastic constants for single-crystal silicon [9]. Ignoring grain-boundary effects, we estimate that $E_{Y}$ for our films is $169 \mathrm{GPa}$ and the fracture strength is in the 2 to $3 \mathrm{GPa}$ range. For comparison, Guckel and co-workers have published a value of $E_{Y}$ for polycrystalline silicon of $22.2 \mathrm{Mpsi}$ (153 GPa) [10].

\section{Polysilicon Material Studies}

An analysis to be published will detail the procedures sketched in the previous paragraph; we provide here only a few features of our studies of polycrystalline silicon to clarify the discussion. Using X-ray diffraction, we have found that the undoped LPCVD polysilicon films grown on PSG at $630^{\circ} \mathrm{C}$ have a preferred orientation that is generally in the (110) direction normal to the substrate. Annealing these films in nitrogen fosters grain growth but does not change their orientation. The polycrystalline-film orientation is actually described in terms of a distribution function derived from analysis of the X-ray diffraction data. This distribution function is used to calculate the effective film properties in terms of single-crystal parameters [8].

\section{CONCLuSIONS}

We have described a technique to build micro-scale movable mechanical pin joints. springs, gears, cranks, and sliders using a silicon microfabrication process. The ability of LPCVD polysilicon to fill undercut regions has been utilized in this research to build new structures including rotating and translating joints, bushings, and sliding elements.

The initial demonstration of the technology has employed polycrystalline silicon for the movable-joint members, but the process is not limited to using this material. The structural members might possibly be made from metals, alloys, and dielectric materials provided that these materials can be freed from their supporting substrate by selective etching of sacrificial (dissolvable) materials. Construction of these new elements gives rise to the need for research on mechanical parameters and properties for design. The process to produce these elements points up the need for further studies of sacrificial-layer etching, LPCVD growth, and remnant stresses in microfabricated systems. At the same time the realization of these structures brings new focus on the brightening prospects for producing microminiature prime movers [11].

The movable micromechanical structures can be batchfabricated into multi-element preassembled mechanisms on a single substrate, or, if desired, they can be freed entirely from their host substrate to be assembled as separate elements. The potential uses for this new technology include the production of miniature ratchets, micro-positioning elements, mechanical logic, tuning elements, optical shutters, micro-valves, micro-pumps, and other mechanisms that have numberless applications in the macroscopic world. The method promises unheralded precision in the construction of miniature mechanical parts and systems with routine control at micrometer dimensions. Their manufacture in the world of micromechanics opens important avenues for further research and development. 


\section{ACKNOWLEDGMENT}

We thank Prof. G. Johnson for valuable discussion on the characterization of polycrystalline films, and $\mathrm{K}$. Voros, R. Hamilton, and the staff of the Berkeley Microfabrication Laboratory for help in experiments and fabrication.

\section{REFERENCES}

[I] L. S. Fan, Y. C. Tai, and R. S. Muller, "Pin-joints, springs, cranks, gears, and other novel micromechanical structures," in Tech. Dig. 4th Int. Conf. Solid-State Sensors and Actuators (Tokyo, June 1987), pp. 849-852 (U.S. patent pending).

[2] K. J. Gabriel, W. S. N. Trimmer, and M. Mehregany, "Micro gear and turbines etched from silicon," in Tech. Dig. 4th Int. Conf. SolidState Sensors and Actuators (Tokyo, June 1987), pp. 853-856.

[3] R. T. Howe and R. S. Muller, "Stress in polycrystalline and amorphous silicon thin films," J. Appl. Phys., vol. 54, pp. 4674-4675, Aug. 1983

[4] H. Guckel, T. Randazzo, and D. W. Burns, "A simple technique for determination of mechanical strain in thin films with applications to polysilicon," J. Appl. Phys., vol. 57, pp. 1671-1675, Mar. I, 1985.

[5] S. Timoshenko, Strength of Materials, 3rd ed. Princeton, NJ: Van Nostrand, 1955

[6] R. P. Kroon and C. C. Davenport, "Spiral springs with small number of turns," J. Franklin Inst., vol. 225, p. 171, 1938.

[7] G. L. Pearson, W. T. Read, and W. L. Feldmann, "Deformation and fracture of small silicon crystals," Acta Metallurgica, vol. 5, pp. 181191, Apr. 1957.

[8] G. C. Johnson, "Acoustoelastic response of polycrystalline aggregates exhibiting transverse isotropy," J. Nondestructive Evaluation, vol. 3, pp. 1-8, 1982

[9] H. J. McSkimin, W. L. Bond, E. Buehler, and G. K. Teal, "Measurement of the elastic constants of silicon single crystals and their thermal coefficients," Phys. Rev. vol. 83, p. 1080, 1951

[10] H. Guckel, D. W. Burns, C. R. Rutigliano, D. K. Showers, and J. Uglow, "Fine grained polysilicon and its application to planar pressure transducers," in Tech. Dig. 4th Int. Conf. Solid-State Sensors and Actuators (Tokyo, June 1987), pp. 277-282.

[11] R. P. Feynman, "There's plenty of room at the bottom," in Miniaturization, H. D. Gilbert, Ed. New York: Reinhold, 1961, pp. 282296.

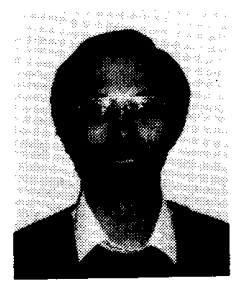

Long-Sheng Fan (M'88) was born in Taiwan, Republic of China, in 1958. He received the B.S. degree in electrical engineering from National Taiwan University, Taipei, Taiwan, Republic of China, in 1974 and the M.S. degree in electrical engineering and computer science from the University of California, Berkeley, in 1984

$\mathrm{He}$ is currently working at the Berkeley Sensor and Actuator Center, Department of Electrical Engineering and Computer Sciences, University of California, Berkeley, on the fundamental as- pects of miniaturization and the actuation of micro-scaled machines and transducers for better interaction between the electronic and nonelectronic worlds. He served in the Chinese Air Force for his ROTC service from 1980 to 1982 . In the 1982-1983 academic year, he received a University of California Regents Fellowship. Since then, he has been supported by the Electronics Research Laboratory at UC, Berkeley, for his research. His major research interests are in novel devices, physics, and materials technology.

Mr. Fan is a member of the American Physics Society.

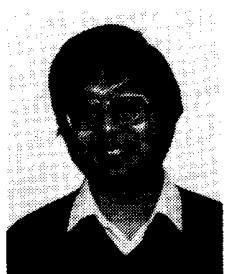

Yu-Chong Tai was born in Taiwan, Republic of China, on May 28, 1959. He received the B.S.E.E. degree from National Taiwan University, Taiwan, in 1981, and the M.S.E.E. degree from the University of California, Berkeley, in 1986. He is currently working toward the Ph.D. degree in the Department of Electrical Engineering and Computer Sciences, University of California. Berkeley.

He served in the Chinese Air Force as a Radio Engineer for his ROTC service from 1981 to 1983. His current research interests are silicon micromachining technology. polysilicon material study, polysilicon micromechanics, and micromechanical integrated sensors such as flow sensors, accelerometers, miniature electric motors, and mechanical logic.

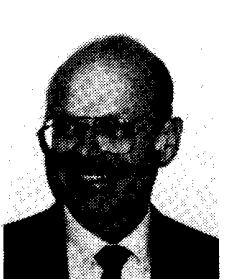

Richard S. Muller (S'57-M'62-SM'70-F'88) received the degree of Mechanical Engineer from the Stevens Institute of Technology, Hoboken, NJ, and the M.S./E.E. and Ph.D. degrees from the California Institute of Technology.

He was a Member of the Technical Staff of the Hughes Aircraft Company and taught at the University of Southern California and the California Institute of Technology before joining the Department of Electrical Engineering and Computer Sciences at the University of California, Berkeley, where he is currently a Professor and Director of the Berkeley Sensor and Actuator Center, a National Science Foundation/industry/university research center. He is the author, together with T. I. Kamins, of Device Electronics for Integrated Circuits, second edition (Wiley, 1986).

Dr. Muller was elected a Fellow of the IEEE for "contributions to solidstate sensors and to education in solid-state electronics" and was awarded a NATO Fellowship (1968-1969) and a Fulbright Senior Research Professorship (1982-1983) at the Technical University, Munich, Germany. He is Chairman of the Sensors Advisory Board for the IEEE Electron Devices Society. He is Chairman of the Steering Committee for the International Conference on Sensors and Actuators, General Chairman of the IEEE 1988 Workshop on Sensors and Actuators, North American Chairman of TRANSDUCERS '89, and General Chairman of TRANSDUCERS '9l. He has also served as Chairman of the Detectors, Sensors, and Displays Committee for the International Electron Devices Meeting and is a member of the editorial boards for Sensors and Actuators and Sensors and Materials. 